Pacific Journal of Mathematics

COBORDISM OBSTRUCTIONS TO FIBERING MANIFOLDS 


\title{
COBORDISM OBSTRUCTIONS TO FIBERING MANIFOLDS OVER SPHERES
}

\author{
SteVEN M. KaHN
}

\begin{abstract}
We consider here the problem introduced by Conner and Floyd of determining necessary and sufficient conditions for a manifold $M$ to be cobordant to a bundle over a given sphere $S^{k}$. Two recent studies by D. F. X. O'Reilly [7] and A. Didierjean [4] presented obstructions to fibering manifolds over spheres in terms of the "top" Stiefel-Whitney classes of $M$. While these conditions were shown by Conner and Floyd [3] and R. L. W. Brown [2] to be sufficient when restricted to the cases of fiberings over $S^{1}$ and $S^{2}$, they are not at all sufficient for guaranteeing the fibering of a cobordism class over a sphere of any higher dimension. This is shown in O'Reilly's study of fiberings over the 4-sphere.

In this paper we exhibit an obstruction to fibering a manifold over a sphere that extends the obstructions mentioned above. We then essentially answer all open questions but one regarding the problem of which cobordism classes can be represented by a bundle over $S^{4}$.
\end{abstract}

1. Introduction. In [3], Conner and Floyd introduced the problem of determining which cobordism classes in $\mathfrak{N}_{*}$ could be represented by a manifold fibered (smoothly) over a given sphere. They showed that if $w_{i}(M)$ denotes the $i$ th Stiefel-Whitney class of a manifold $M$, then a class $\omega \in \mathfrak{R}_{n}$ fibers over $S^{1}$ (i.e. $\omega$ contains a representative that fibers over $S^{1}$ ) if and only if the Stiefel-Whitney number $w_{n}(\omega)=0$. Extending the study, R. L. W.Brown [2] showed that a class $\omega \in \mathfrak{N}_{n}$ fibers over $S^{2}$ if and only if the Stiefel-Whitney number $w_{n}(\omega)=0$ if $n$ is even and $w_{2} w_{n-2}(\omega)=0$ if $n$ is odd. Subsequent investigations have considered fiberings over a variety of manifolds, yielding complete solutions in a number of cases. But R. E. Stong [8] observed that spheres, aside from having a natural importance, actually play a key role here. He showed

(1.1) If a class $\omega \in \mathfrak{R}_{n}$ fibers over $S^{k}$, then $\omega$ fibers over any manifold $N^{q}$ with $q \leq k$.

At present, for fiberings over spheres of any higher dimension than two, there are only partial results. In solving the $S^{2}$ problem, Brown exhibited a general necessary condition [2, Prop. 2.1] for a manifold to fiber over a sphere $S^{k}$ that is based on the Brown-Peterson relations among characteristic classes. Using it, he derived the Stiefel-Whitney number obstructions mentioned above. D. F. X. O'Reilly [7] (see also A. 
Didierjean [4]) generalized those obstructions as follows:

(1.2) If $M^{n}$ fibers over $S^{2 k}$, then all Stiefel-Whitney numbers divisible by

$$
\begin{cases}w_{n}, w_{n-1}, \ldots, w_{n-2 k+1} & \text { for } n \text { even } \\ w_{n}, w_{n-1}, \ldots, w_{n-2 k} & \text { for } n \text { odd }\end{cases}
$$

are zero.

What is significant, is that although these conditions turn out to be sufficient for a cobordism class to fiber over $S^{2}$, they are far from sufficient for guaranteeing the fibering of a class over a sphere of any higher dimension. This is made clear in O'Reilly's study of fiberings over the 4-sphere. The conditions given by (1.2) are shown to leave the fibering status of a good number of classes unaccounted for.

The purpose of this paper is to somewhat expand Brown's condition (involving the Brown-Peterson relations) and then more importantly, demonstrate its use; not as a device for obtaining obstructions in terms of Stiefel-Whitney numbers but actually as an obstruction itself to fibering cobordism classes over spheres. With this point of view we are able to extend the results of O'Reilly and Didierjean, essentially answering all open questions but one, regarding the problem of which cobordism classes fiber over $S^{4}$.

Throughout this paper we will use no notation to differentiate between a manifold and its cobordism class. Also all cohomology will be with $\mathbf{Z}_{2}$ coefficients.

2. Main results. Given a (smooth, closed) manifold $M^{n}$, let $\tau_{M}: M^{n} \rightarrow B O$ classify the stable tangent bundle of $M^{n}$ and let the $(\bmod 2)$ Steenrod algebra $\mathcal{Q}$ act on the right of $H^{*}(B O)$ and $H^{*}(M)$ as in [1]. Before stating our results we briefly recall the Brown-Peterson relations for reference [1].

(2.1) (Brown-Peterson): $\bigcap_{M^{n}} \operatorname{ker} \tau_{M}^{*}=\sum H^{s}(B O) \mathrm{Sq}^{t}, 2 t>n-s$.

Now, using Didierjean's notation let

$$
I_{0}^{k}\left(M^{n}\right)=\left\{\alpha \in H^{k}(B O) \mid \text { if } k \leq n, \tau_{M}^{*}(\alpha \cdot \beta)=0 \forall \beta \in H^{n-k}(B O)\right\} .
$$

Noting that $I_{0}^{*}\left(M^{n}\right)$ depends only on the cobordism class $\omega$ of $M^{n}$ let

$$
A_{0}^{*}(\omega)=H^{*}(B O) / I_{0}^{*}(\omega) .
$$


THEOREM 1. If $\omega \in \mathfrak{R}_{n}$ fibers over $S^{k}$, then $\left[\operatorname{Sq}^{r}\left(H^{s}(B O) \mathrm{Sq}^{t}\right)\right]=0$ $\in A_{0}^{*}(\omega)$ and $\left[\left(\sum H^{s}(B O) \mathrm{Sq}^{t}\right)^{2}\right]=0 \in A_{0}^{*}(\omega)$ for $2 t>n-k-s$ and $r>s+t-k$.

Proof. Let $p: M^{n} \rightarrow S^{k}$ be a fibration with fiber $F^{n-k}$ and with $M^{n}$ representing the cobordism class $\omega$. That $\left[\mathrm{Sq}^{r}\left(H^{s}(B O) \mathrm{Sq}^{t}\right)\right]=0$ is essentially Brown's condition [2, Prop. 2.1]. That $\left[\left(\sum H^{s}(B O) \mathrm{Sq}^{t}\right)^{2}\right]=0$ will follow from the same reasoning. As in [2], we consider the exact sequence

$$
\rightarrow H^{*}(M, F) \stackrel{j^{*}}{\rightarrow} H^{*}(M) \stackrel{i^{*}}{\rightarrow} H^{*}(F) \rightarrow
$$

and note that by excision

$$
H^{*}(M, F)=H^{*}\left(M, F \times D^{k}\right)=H^{*}\left(F \times D^{k}, F \times S^{k-1}\right)
$$

where $D^{k}$ is the $k$-ball. Then

$$
H^{*}(M, F)=H^{*-k}(F) \otimes H^{k}\left(D^{k}, S^{k-1}\right) .
$$

Now

$$
i^{*} \tau_{M}^{*} H^{s}(B O) \mathrm{Sq}^{t}=\tau_{F}^{*} H^{s}(B O) \mathrm{Sq}^{t}=0 \text { for } 2 t>n-k-s
$$

by (2.1). Let $u, v \in \Sigma H^{s}(B O) \mathrm{Sq}^{t}, 2 t>n-k-s$. Then by (2.2) and (2.3), $\tau_{M}^{*} u=j^{*}(x \otimes a)$ and $\tau_{M}^{*} v=j^{*}(y \otimes a)$ for some $x, y \in H^{*}(F)$, $a \in H^{k}\left(D^{k}, S^{k-1}\right)$ being the generator. Hence $\tau_{M}^{*}(u v)=\tau_{M}^{*} u \cdot \tau_{M}^{*} v=0$ since $a^{2}=0$ and so $\left[\left(\sum H^{s}(B O) \mathrm{Sq}^{t}\right)^{2}\right]=0 \in A_{0}^{*}(\omega)$.

Interestingly, having added to Brown's original condition, we now show that in practice Theorem 1 can actually be simplified back again.

Proposition 2.4. For any class $\omega \in \mathfrak{N}_{n}$ and $k \leq n$,

$$
\left[\operatorname{Sq}^{r}\left(H^{s}(B O) \mathrm{Sq}^{t}\right)\right]=0 \in A_{0}^{*}(\omega) \Leftrightarrow\left[\left(\sum H^{s}(B O) \mathrm{Sq}^{t}\right)^{2}\right]=0 \in A_{0}^{*}(\omega)
$$

for $2 t>n-k-s$ and $r>s+t-k$.

Proof. Let $M^{n}$ represent $\omega$.

$(\Leftarrow)$ Let $u \in H^{s}(B O)$. By definition of the right action of $\mathcal{Q}$ on $H^{*}(M)$, we have for any $\beta \in H^{n-r-s-t}(B O)$

$$
\left\langle\tau_{M}^{*}\left(\beta \cdot \mathrm{Sq}^{r}\left(u \mathrm{Sq}^{t}\right)\right),\left[M^{n}\right]\right\rangle=\left\langle\tau_{M}^{*}\left(\beta \mathrm{Sq}^{r} \cdot u \mathrm{Sq}^{t}\right),\left[M^{n}\right]\right\rangle .
$$

Now since $r>s+t-k$, we get that $2 r>n-k-(n-r-s-t)$ and so $\beta \mathrm{Sq}^{r} \in \sum H^{s}(B O) \mathrm{Sq}^{t}$ for $2 t>n-k-s$. Therefore by (2.5),

$$
\left[\left(\sum H^{s}(B O) \mathrm{Sq}^{t}\right)^{2}\right]=0 \Rightarrow\left[\mathrm{Sq}^{r}\left(H^{s}(B O) \mathrm{Sq}^{t}\right)\right]=0
$$


$(\Rightarrow)$ Now suppose $\left[\mathrm{Sq}^{r}\left(H^{s}(B O) \mathrm{Sq}^{t}\right)\right]=0$. Let $x=u \mathrm{Sq}^{J} \in$ $H^{\prime}(B O) \mathrm{Sq}^{j}$ and $y=v \mathrm{Sq}^{m} \in H^{l}(B O) \mathrm{Sq}^{m}$ with $2 j>n-k-i$ and $2 m>$ $n-k-l$ (i.e. $\left.x, y \in \Sigma H^{s}(B O) \mathrm{Sq}^{t}, 2 t>n-k-s\right)$. To show that $[x \cdot y]$ $=0 \in A_{0}^{*}(\omega)$, let $\gamma \in H^{n-(l+j+l+m)}(B O)$. Since $\sum H^{s}(B O) \mathrm{Sq}^{t}, 2 t>n-$ $k-s$ is an ideal (see (2.1)), we have that $\gamma \cdot v \mathrm{Sq}^{m} \in \sum H^{s}(B O) \mathrm{Sq}^{t}, 2 t>$ $n-k-s$. Thus $\tau_{M}^{*}\left(\gamma \cdot v \mathrm{Sq}^{m} \cdot u \mathrm{Sq}^{j}\right)=\tau_{M}^{*}\left(\beta \mathrm{Sq}^{r} \cdot u \mathrm{Sq}^{j}\right)$ for some $\beta \mathrm{Sq}^{r} \in H^{n-r-i-j}(B O) \mathrm{Sq}^{r}$ with $2 r>n-k-(n-r-i-j)$. By (2.5)

$$
\left\langle\tau_{M}^{*}\left(\gamma \cdot v \mathrm{Sq}^{m} \cdot u \mathrm{Sq}^{j}\right),[M]\right\rangle=\left\langle\tau_{M}^{*}\left(\beta \cdot \mathrm{Sq}^{r}\left(u S q^{j}\right)\right),[M]\right\rangle
$$

and since $r>i+j-k$ the result follows.

The fact that the conditions given by Theorem 1 prove to be sufficient for a class in $\mathfrak{R}_{*}$ to fiber over $S^{1}$ or $S^{2}$ can be expressed as follows. All characteristic number obstructions to fibering a manifold over $S^{1}$ or $S^{2}$ come from those characteristic classes of the total space that by the Brown-Peterson relations restrict to zero on the fiber. In [5] however, it was observed that all obstructions to fibering a complex manifold over $S^{3}$ (or $S^{4}$ ), with the exception of a signature condition, actually arise simply from $\mathrm{Wu}$ classes $v_{i}$ pulling back to zero on the fiber. Indeed, this is really the case with the Conner-Floyd and Brown results as well. Noting that $w_{2 n}\left(M^{2 n}\right)=\operatorname{Sq}^{n} v_{n}\left(M^{2 n}\right)$ and $w_{2 n-1}\left(M^{2 n+1}\right)=\operatorname{Sq}^{n-1} v_{n}\left(M^{2 n+1}\right)$ by the $\mathrm{Wu}$ formula, we see that consideration of the full range of Brown-Peterson relations is unnecessary in the $S^{1}$ and $S^{2}$ cases. The Wu classes alone produce all sufficient fibering conditions. Conceivably, for any $\omega \in \mathfrak{N}_{n}$ and $k \leq n,\left[\mathrm{Sq}^{r}\left(H^{s}(B O) \mathrm{Sq}^{t}\right)\right]=0 \in A_{0}^{*}(\omega)$ for $2 t>n-k-s, r>s+$ $t-k$ if and only if $\left[\mathrm{Sq}^{r} v_{t}\right]=0 \in A_{0}^{*}(\omega)$ for $2 t>n-k, r>t-k$. (Note that $v_{t}=(1) \mathrm{Sq}^{t} \in H^{0}(B O) \mathrm{Sq}^{t}$.)

Turning to the problem of fiberings over $S^{4}$, the key role is again played by the $\mathrm{Wu}$ classes.

Let $X_{4}, X_{5}$ and $X_{6}$ be indecomposables in $\Re_{*}$ of dimensions 4,5 and 6 respectively.

THEOREM 2. (i) A class $\omega \in \mathfrak{R}_{2 n+1}$ fibers over $S^{4}$ if and only if

$$
\left[\mathrm{Sq}^{r} v_{t}\right]=0 \in A_{0}^{*}(\omega) \text { for } t=n, n-1, r>t-4 \text {. }
$$

(ii) If $\omega \in \mathfrak{R}_{2 n}$ is such that $\left[\mathrm{Sq}^{r} v_{t}\right]=0 \in A_{0}^{*}(\omega)$ for $t=n, n-1$, $r>t-4$, then $\omega=Y+a \mathbf{R} P(2)^{n-5}\left(X_{4} X_{6}+X_{5}^{2}\right)$ where $Y$ fibers over $S^{4}$ and $a \in \mathbf{Z}_{2}$.

Remark. For $\omega=X_{4} X_{6}+X_{5}^{2} \in \mathfrak{R}_{10},\left[\mathrm{Sq}^{r} v_{t}\right]=0 \in A_{0}^{*}(\omega)$ for $t=4,5, r>t-4$. In fact, $\left[\operatorname{Sq}^{r}\left(H^{s}(B O)\right) \mathrm{Sq}^{t}\right]=0 \in A_{0}^{*}(\omega)$. It is still 
unknown however whether or not there exists a representative of $\omega$ that fibers over $S^{4}$.

The proof of Theorem 2 essentially takes up the remainder of the paper. The key to the proof is in $\S 3$ where Theorem 1 is applied. Specific obstructions, going beyond those given in [7] and [4], are exhibited and are shown to account for a number of families of classes in $\Re_{*}$ not fibering over $S^{4}$. In $\$ 4$ we tie up the loose ends through actual construction of fibrations and formally complete the proof. In $\S 5$ we present a result about fiberings over $S^{3}$.

3. Obstructions. We begin this section by recalling results of Brown and O'Reilly.

Proposition 3.1 ( Brown).

(i) There exist indecomposables $X_{i} \in \mathfrak{R}_{i}$ fibered over $S^{2}, i \neq 2^{s}-1$, and $i \neq 2,5$.

(ii) If $\omega \in \mathfrak{R}_{n}$ fibers over $S^{2}$, then $\omega^{2}$ fibers over $S^{4}$.

Proposition 3.2 (O'Reilly).

(i) There exist indecomposables $X_{i} \in \mathfrak{A}_{i}$ fibered over $S^{4}, i \neq 2^{s}-1$, and $i \neq 2,4,5,6,11$.

(ii) The class $X_{4} X_{5} X_{6}+X_{5}^{3}$ fibers over $S^{4}$.

Throughout the rest of this paper we will let $X_{i} \in \mathfrak{N}_{i}$ denote O'Reilly's indecomposables for $i \neq 2,4,5,6,11$ while denoting Brown's indecomposables (fibered over $S^{2}$ ) for $i=4,6$ and 11 .

O'Reilly showed that no indecomposable $X_{11}$ could fiber over $S^{4}$ and proved the same thing for the classes $X_{4} X_{6}$ and $X_{5}^{2}$. He conjectured that $X_{2} X_{11}, X_{2}^{2} X_{11}$ and $X_{5}^{3}$ also do not fiber over $S^{4}$ even though all obstructions given by (1.2) vanish on these classes. The following two propositions prove these conjectures while extending O'Reilly's results in general.

Proposition 3.3. For any non-zero linear combination

$$
\begin{aligned}
\omega= & a_{1} X_{2}^{n-2} X^{5}+a_{2} X_{2}^{n-4} X_{4} X_{5}+a_{3} X_{2}^{n-5} X_{5} X_{6}+a_{4} X_{2}^{n-5} X_{11} \\
& +a_{5} X_{2}^{n-7} X_{4} X_{5} X_{6}+a_{6} X_{2}^{n-7} X_{5}^{3}, \quad a_{\imath} \in \mathbf{Z}_{2},
\end{aligned}
$$

in $\mathfrak{\Re}_{2 n+1}$, with the exception of $\omega=X_{2}^{n-7}\left(X_{4} X_{5} X_{6}+X_{5}^{3}\right),\left[\mathrm{Sq}^{r} v_{t}\right]$ for $t=n, n-1, r>t-4$ is non-zero in $A_{0}^{*}(\omega)$. And no such combination fibers over $S^{4}$. 
Proof. If $a_{1}=1$, Brown's $S^{2}$ result shows that $w_{2} w_{2 n-1}(\omega) \neq 0$. Since $w_{2 n-1}\left(M^{2 n+1}\right)=\operatorname{Sq}^{n-1} v_{n}\left(M^{2 n+1}\right)$, the result follows. Thus we need only look at the case where $a_{1}=0$. Consider the following chart obtained via the product formulas for the Stiefel-Whitney and $\mathrm{Wu}$ classes:

\begin{tabular}{l|c|c|c|c} 
& $X_{2}^{n-4} X_{4} X_{5}$ & $X_{2}^{n-5} X_{5} X_{6}$ & $X_{2}^{n-5} X_{11}$ & $X_{2}^{n-7} X_{5}^{3}$ \\
\hline$w_{2}^{3} \mathrm{Sq}^{n-4} v_{n-1}$ & $n$ & $n+1$ & 1 & 0 \\
$w_{6} \mathrm{Sq}^{n-4} v_{n-1}$ & $n$ & $\left(\begin{array}{c}n-2 \\
2\end{array}\right)$ & 0 & 1 \\
$w_{1} w_{3} \mathrm{Sq}^{n-3} v_{n}$ & 1 & 1 & 0 & 0 \\
$w_{4} \mathrm{Sq}^{n-3} v_{n}$ & 1 & 0 & 0 & 0
\end{tabular}

Clearly the above matrix is non-singular. Furthermore, since $X_{4} X_{5} X_{6}+X_{5}^{3}$ fibers over $S^{4}$, Theorem 1 implies that

$$
\left[\mathrm{Sq}^{r} v_{t}\right]=0 \in A_{0}^{*}\left(X_{2}^{n-7}\left(X_{4} X_{5} X_{6}+X_{5}^{3}\right)\right)
$$

and so we need not consider a column for $X_{2}^{n-7} X_{4} X_{5} X_{6}$. The result follows.

Proposition 3.4. For any non-zero linear combination

$$
\begin{aligned}
\omega= & a_{1} X_{2}^{n}+a_{2} X_{2}^{n-2} X_{4}+a_{3} X_{2}^{n-3} X_{6}+a_{4} X_{2}^{n-5} X_{4} X_{6} \\
& +a_{5} X_{2}^{n-5} X_{5}^{2}, \quad a_{i} \in \mathbf{Z}_{2},
\end{aligned}
$$

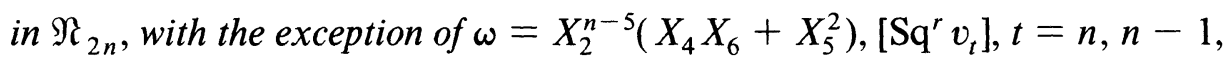
$r>t-4$ is non-zero in $A_{0}^{*}(\omega)$. And no such combination fibers over $S^{4}$.

Proof. Stong [8, Prop. 7.2] showed that the classes $X_{2}^{n}, X_{2}^{n-2} X_{4}$ and $X_{2}^{n-3} X_{6}$ are distinguished by characteristic numbers involving the StiefelWhitney classes $w_{2 n}, w_{2 n-1}, w_{2 n-2}$ and $w_{2 n-3}$. By the Wu formula,

$$
\begin{gathered}
w_{2 n}\left(M^{2 n}\right)=\mathrm{Sq}^{n} v_{n}\left(M^{2 n}\right), \quad w_{2 n-1}\left(M^{2 n}\right)=\mathrm{Sq}^{n-1} v_{n}\left(M^{2 n}\right), \\
w_{2 n-2}\left(M^{2 n}\right)=\mathrm{Sq}^{n-2} v_{n}+\mathrm{Sq}^{n-1} v_{n-1}\left(M^{2 n}\right)
\end{gathered}
$$

and

$$
w_{2 n-3}\left(M^{2 n}\right)=\mathrm{Sq}^{n-3} v_{n}+\mathrm{Sq}^{n-2} v_{n-1}\left(M^{2 n}\right) .
$$

Thus for any class $\omega$, if $\left[\mathrm{Sq}^{r} v_{t}\right]=0$ for $t=n, n-1, r>t-4$, then $\left[w_{2 n}\right]=\left[w_{2 n-1}\right]=\left[w_{2 n-2}\right]=\left[w_{2 n-3}\right]=0 \in A_{0}^{*}(\omega)$. Since all numbers involving $w_{2 n}, w_{2 n-1}, w_{2 n-2}$ and $w_{2 n-3}$ vanish for both $X_{2}^{n-5} X_{4} X_{6}$ and $X_{2}^{n-5} X_{5}^{2}$ we see that the only combination we must account for is 
$\omega=a_{4} X_{2}^{n-5} X_{4} X_{6}+a_{5} X_{2}^{n-5} X_{5}^{2}$. An easy calculation using the product formula for the Stiefel-Whitney and Wu classes shows that

$$
w_{4} \mathrm{Sq}^{n-3} v_{n-1}\left(X_{2}^{n-5} X_{4} X_{6}\right)=w_{4} \mathrm{Sq}^{n-3} v_{n-1}\left(X_{2}^{n-5} X_{5}^{2}\right) \neq 0
$$

and so if $a_{4} \neq a_{5}$, then [ $\left.\mathrm{Sq}^{r} v_{t}\right] t=n, n-1, r>t-4$ is non-zero in $A_{0}^{*}\left(a_{4} X_{2}^{n-5} X_{4} X_{6}+a_{5} X_{2}^{n-5} X_{5}^{2}\right)$. The result follows.

4. Some fibrations over $\mathbf{S}^{4}$. Since the classes in $\mathfrak{R}_{*}$ which fiber over $S^{4}$ form an ideal, the results of $\S 3$ combined with Propositions 3.1 and 3.2 leave only a few classes still unaccounted for. In this section we show that the classes $X_{4} X_{5}^{2}, X_{4} X_{11}, X_{5}^{2} X_{6}, X_{5} X_{11}$ and $X_{6} X_{11}$ all fiber over $S^{4}$. First some preliminaries.

Let $\xi$ be an $n$-dimensional vector bundle over a manifold $B$. The real projective bundle $\mathbf{R} P(\xi)$ is the manifold consisting of all lines in the fibers of $\xi$. $\mathbf{R} P(\xi)$ fibers over $B$, say $p: \mathbf{R} P(\xi) \rightarrow B$ (with fiber $\mathbf{R} P(n-1)$ ), and supports a canonical line bundle $\eta$.

Let $c=w_{1}(\eta) \in H^{1}(\mathbf{R} P(\xi))$. Then $H^{*}(\mathbf{R} P(\xi))$ is a free $H^{*}(B)$ module on generators $1, c, \ldots, c^{n-1}$ with the relation

$$
\sum_{i=0}^{n} c^{l} p^{*}\left(w_{n-i}(\xi)\right)=0 .
$$

The tangent bundle of $\mathbf{R} P(\xi)$ is given by

$$
\tau(\mathbf{R} P(\xi)) \oplus \theta^{1}=p^{*} \tau(B) \oplus\left(p^{*} \xi \otimes \eta\right)
$$

where $\theta^{1}$ denotes the trivial line bundle.

For convenience, we will from now on omit the " $p$ " from our notation and will let $\eta$ once and for all denote the appropriate canonical line bundle over whatever projective bundle we may be dealing with. We let $\gamma$ denote the quaternionic line bundle over $S^{4}=\mathbf{H} P(1)(\gamma$ is a real 4-bundle) and we let $\theta^{n}$ denote the trivial $n$-bundle.

Finally, we recall some facts (see [6]) about the so-called $s$ numbers of a manifold. If the total Stiefel-Whitney class $w$ of a bundle $E$ over $M$ is given by $w(E)=\left(1+t_{1}\right)\left(1+t_{2}\right) \cdots\left(1+t_{k}\right)$ for $t_{l} \in H^{i}(M)$, then for any partition $I=i_{1}, i_{2}, \ldots, i_{r}$ of $k$, the polynomial $s_{I}$ in the Stiefel-Whitney classes of $E$ is defined by

$$
s_{I}\left(w_{1}, \ldots, w_{k}\right)=\sum t_{1}^{i_{1}} \cdots t_{r}^{i_{r}}
$$

the sum ranging over all permutations of $t_{1}, \ldots, t_{k}$. For any manifold $M$, $s_{I}(M)=s_{I}(\tau(M))$. We also note

$$
s_{I}\left(E_{1} \oplus E_{2}\right)=\sum_{J K=I} s_{J}\left(E_{1}\right) s_{K}\left(E_{2}\right)
$$


and for any indecomposables $X_{i} \in \mathfrak{N}_{i}, i \neq 2^{s}-1$,

$$
s_{I}\left(X_{i_{1}} X_{i_{2}} \cdots X_{i_{r}}\right)=0 \text { if } I \text { is not a refinement of } J=i_{1}, \ldots, i_{r} .
$$

End of preliminaries.

The following lemmas are needed for future calculations.

LEMMA 4.6. For Brown's indecomposables $X_{4}=\mathbf{R} P\left(\lambda \oplus \theta^{1}\right)$ over $S^{2}$ and $X_{6}=\mathbf{R} P\left(\lambda \oplus \theta^{3}\right)$ over $S^{2}, \lambda$ being the canonical complex line bundle over $S^{2}=\mathbf{C P}(1), s_{2,2}\left(X_{4}\right)=1$ and $s_{2,4}\left(X_{6}\right)=s_{2,2,2}\left(X_{6}\right)=0$.

Proof. By (4.2), $w\left(X_{4}\right)=\left((1+c)^{2}+a\right)(1+c)$ while $w\left(X_{6}\right)=$ $\left((1+c)^{2}+a\right)(1+c)^{3}$ where $a \in H^{2}\left(S^{2}\right)$ is the generator and $c=$ $w_{1}(\eta) \in H^{1}\left(\mathbf{R} P\left(\lambda \oplus \theta^{1}\right)\right) \quad\left(\right.$ resp. $\left.H^{1}\left(\mathbf{R} P\left(\lambda \oplus \theta^{3}\right)\right)\right)$. Now, $s_{2,2}\left(X_{4}\right)=$ $w_{2}^{2}\left(X_{4}\right)=1$ (using (4.1)). Similarly,

$$
s_{2,4}\left(X_{6}\right)=s_{1,2}^{2}\left(X_{6}\right)=\left(w_{1} w_{2}+w_{3}\right)^{2}\left(X_{6}\right)=0
$$

while

$$
s_{2,2,2}\left(X_{6}\right)=s_{1,1,1}^{2}\left(X_{6}\right)=w_{3}^{2}\left(X_{6}\right)=0 \text {. }
$$

LEMMA 4.7. For the indecomposable $X_{10}=\mathbf{R} P\left(\gamma \oplus \theta^{3}\right)$ over $S^{4}$, $s_{5,5}\left(X_{10}\right)=1$.

Proof. By (4.2), $w\left(X_{10}\right)=\left((1+c)^{4}+a\right)\left(1+c^{3}\right)$ where $c=w_{1}(\eta)$ and $a \in H^{4}\left(S^{4}\right)$ is the generator. Since the only non-zero Stiefel-Whitney class of $\gamma \otimes \eta$ is $w_{4}(\gamma \otimes \eta)=c^{4}+a$, any $s$-class of $\gamma \otimes \eta$ with dimension not a multiple of 4 vanishes. By (4.4) then, $s_{5,5}\left(X_{10}\right)=s_{5,5}\left(\theta^{3} \otimes \eta\right)$. But $s_{5,5}\left(\theta^{3} \otimes \eta\right)=c_{1}^{5} c_{2}^{5}+c_{1}^{5} c_{3}^{5}+c_{2}^{5} c_{3}^{5}$. The result follows from (4.1).

LEMMA 4.8. For the indecomposable $X_{11}=\mathbf{R} P(\lambda \oplus \tau \mathbf{R} P(4))$ over $S^{2} \times \mathbf{R} P(4), \lambda$ being the canonical complex line bundle over $S^{2}=\mathbf{C P}(1)$, $s_{5,6}\left(X_{11}\right)=s_{2,9}\left(X_{11}\right)=s_{5,2,4}\left(X_{11}\right)=s_{5,2,2,2}\left(X_{11}\right)=0$.

Proof. By (4.2), $w\left(X_{11}\right)=(1+\alpha)^{5}\left((1+c)^{2}+a\right)(1+c+\alpha)^{5}$ where $\alpha$ and $a$ are the pullbacks of the generators of $H^{1}(\mathbf{R} P(4))$ and $H^{2}\left(S^{2}\right)$ respectively and $c=w_{1}(\eta)$. Write $\left((1+c)^{2}+a\right)=\left(1+t_{1}\right)\left(1+t_{2}\right)$ and note that $t_{1}=t_{2}$ and $t_{1} t_{2}=c^{2}+a$. The rest of the proof is a routine calculation using (4.3) and (4.1).

LEMMA 4.9. For the indecomposable $X_{12}=\mathbf{R} P\left(\gamma \oplus \theta^{5}\right)$ over $S^{4}$, $s_{10,2}\left(X_{12}\right)=s_{5,5,2}\left(X_{12}\right)=0$. 
Proof. By (4.2), $w\left(X_{12}\right)=\left((1+c)^{4}+a\right)(1+c)^{5}$. Since the only non-zero Stiefel-Whitney class of $\gamma \otimes \eta$ is $w_{4}(\gamma \otimes \eta)=c^{4}+a$, any $s$-class with dimension not a multiple of 4 vanishes. Furthermore $w_{4}^{3}(\gamma \otimes \eta)=c^{12}$ $+c^{8} a=0$ by (4.1), so any $s$-class of dimension 12 vanishes as well. Then by (4.4), $s_{10,2}\left(X_{12}\right)=s_{10,2}\left(\theta^{5} \otimes \eta\right)$ and $s_{5,5,2}\left(X_{12}\right)=s_{5,5,2}\left(\theta^{5} \otimes \eta\right)$. The result follows from (4.3) and (4.1).

We now turn to the main results of the section.

Proposition 4.10. The class $X_{4} X_{5}^{2}$ fibers over $S^{4}$.

Proof. Let

$$
\begin{gathered}
M^{14}=\mathbf{R} P\left(\eta_{1} \oplus \theta^{5}\right) \\
\downarrow \\
\mathbf{R} P\left(\gamma \oplus \theta^{2}\right) \\
\downarrow \\
S^{4}
\end{gathered}
$$

Since $M^{14}$ fibers over $S^{4}$, Theorem 1 guarantees that all characteristic numbers of $M$ divisible by $\mathrm{Sq}^{r} v_{t}, t=6,7, r>t-4$ are zero in $A_{0}^{*}\left(X_{4} X_{5}^{2}\right)$. Therefore, applying Propositions 3.1, 3.2 and 3.4, we have that the cobordism class of $M^{14}$ is given by

$$
M^{14}=a_{1} X_{4} X_{5}^{2}+a_{2} X_{2}^{2}\left(X_{4} X_{6}+X_{5}^{2}\right)+Y, a_{k} \in \mathbf{Z}_{2},
$$

where $Y$ fibers over $S^{4}$. Now by (4.2),

$$
w\left(M^{14}\right)=\left((1+c)^{4}+a\right)(1+c)^{2}(1+d+c)(1+d)^{5}
$$

where $c=w_{1}\left(\eta_{1}\right)$ and $d=w_{1}\left(\eta_{2}\right)\left(\eta_{2}\right.$ being the canonical line bundle over $\left.\mathbf{R} P\left(\eta_{1} \oplus \theta^{5}\right)\right)$. As in the proof of Lemma 4.9, any s-class of $\gamma \otimes \eta_{1}$ with dimension not a multiple of 4 or with dimension 12 vanishes. In addition, for any bundle $E, s_{4}(E)=w_{1}^{4}(E)$ and $s_{2,2}(E)=w_{2}^{2}(E)$, so $s_{4}\left(\gamma \otimes \eta_{1}\right)$ and $s_{2,2}\left(\gamma \otimes \eta_{1}\right)$ vanish as well. Applying (4.4) and (4.3) as in the previous proofs, we arrive at the following $s$-numbers for $M: s_{14}=0, s_{10,4}=1$, $s_{9,5}=0, s_{5,5,4}=0, s_{10,2,2}=1$ and $s_{5,5,2,2}=0$. By (4.5) and Lemmas 4.6, 4.7 and 4.9 , it must be that

$$
M^{14}=1 \cdot X_{4} X_{5}^{2}+0 \cdot X_{2}^{2}\left(X_{4} X_{6}+X_{5}^{2}\right)+Y
$$

and so by adding $Y$ to both sides we get that $X_{4} X_{5}^{2}$ fibers over $S^{4}$.

Proposition 4.11. The class $X_{4} X_{11}$ fibers over $S^{4}$. 
Proof. Let

$$
\begin{gathered}
M^{15}=\mathbf{R} P\left(\eta_{1} \oplus \theta^{5}\right) \\
\downarrow \\
\mathbf{R} P\left(\gamma \oplus \lambda \oplus \theta^{1}\right) \\
\downarrow \\
S^{4} \times S^{1}
\end{gathered}
$$

where $\lambda$ is the pullback of the canonical line bundle over $S^{1}$. Noting that $\left[\mathrm{Sq}^{r} v_{t}\right]=0 \in A_{0}^{*}\left(X_{4} X_{11}\right)$ (for $t=6,7 r>t-4$ ), we invoke the results of section 3, as in the proof of Proposition 4.10, to see that $M^{15}=a X_{4} X_{11}+$ $Y$ where $Y$ fibers over $S^{4}$. Now by (4.2)

$$
w\left(M^{15}\right)=\left((1+c)^{4}+a\right)(1+c+\alpha)(1+c)(1+d+c)(1+d)^{5}
$$

where $\alpha$ is the pullback of the generator of $H^{1}\left(S^{1}\right)$. Again as in the previous proofs we can ignore any contribution from $\gamma \otimes \eta_{1}$, obtaining $s_{15}\left(M^{15}\right)=0$ and $s_{4,11}\left(M^{15}\right)=1$. Then $M^{15}=1 \cdot X_{4} X_{11}+Y$ and so $X_{4} X_{11}$ fibers over $S^{4}$.

Proposition 4.12. The classes $X_{5}^{2} X_{6}$ and $X_{5} X_{11}$ fiber over $S^{4}$.

Proof. Let

$$
\begin{array}{ccc}
M_{1}^{16}=\mathbf{R} P\left(\eta_{1} \oplus \theta^{3}\right) & \text { and let } & M_{2}^{16}=\mathbf{R} P\left(\eta_{1} \oplus \theta^{5}\right) \\
\downarrow & \mathbf{R} P\left(\gamma \oplus \lambda \oplus \theta^{3}\right) & \mathbf{R} P\left(\gamma \oplus \lambda \oplus \theta^{1}\right) \\
\downarrow & \downarrow \\
S^{4} \times \mathbf{R} P(2) & S^{4} \times \mathbf{R} P(2)
\end{array}
$$

where $\lambda$ is the pullback of the canonical line bundle over $\mathbf{R} P(2)$. As before, we see that

$$
M_{i}^{16}=a_{i 1} X_{5}^{2} X_{6}+a_{i 2} X_{5} X_{11}+a_{i 3} X_{2}^{3}\left(X_{4} X_{6}+X_{5}^{2}\right)+Y_{i}, \quad i=1,2,
$$

where $Y_{i}$ fibers over $S^{4}$ and $a_{i j} \in \mathbf{Z}_{2}$. By (4.2)

$$
\begin{aligned}
w\left(M_{1}^{16}\right)= & (1+\alpha)^{3}\left((1+c)^{4}+a\right)(1+c+\alpha)(1+c)^{3} \\
& \times(1+d+c)(1+d)^{3}, \\
w\left(M_{2}^{16}\right)= & (1+\alpha)^{3}\left((1+c)^{4}+a\right)(1+c+\alpha)(1+c) \\
& \times(1+d+c)(1+d)^{5}
\end{aligned}
$$


where $\alpha$ is the pullback of the generator of $H^{1}(\mathbf{R} P(2))$. Now by techniques demonstrated earlier, we obtain the following chart of $s$-numbers:

\begin{tabular}{c|c|c|c|c|c|c|c|c|c|c} 
& $s_{16}$ & $s_{5,11}$ & $s_{10,6}$ & $s_{2,14}$ & $s_{5,5,6}$ & $s_{2,5,9}$ & $s_{2,4,10}$ & $s_{5,5,2,4}$ & $s_{10,2,2,2}$ & $s_{5,5,2,2,2}$ \\
\hline$M_{1}^{16}$ & 0 & 0 & 0 & 0 & 1 & 0 & 0 & 0 & 0 & 0 \\
$M_{2}^{16}$ & 0 & 1 & 1 & 0 & 0 & 0 & 0 & 0 & 0 & 0
\end{tabular}

Then by (4.5) and Lemmas 4.6-4.9, $M_{1}^{16}=X_{5}^{2} X_{6}+Y_{1}$ and $M_{2}^{16}=X_{5}^{2} X_{6}$ $+X_{5} X_{11}+Y_{2}$. The result follows by addition.

Proposition 4.13. The class $X_{6} X_{11}$ fibers over $S^{4}$.

Proof. Let

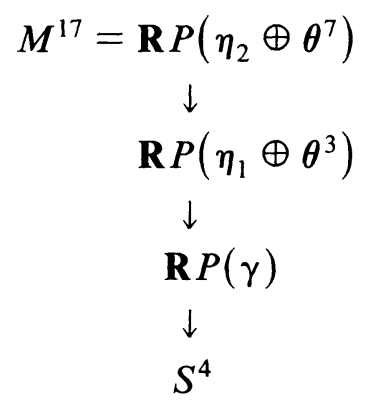

Routine calculation shows that $s_{17}\left(M^{17}\right)=1$ and $s_{6,11}\left(M^{17}\right)=0$, while for the indecomposable $X_{17}, s_{17}\left(X_{17}\right)=1$ and $s_{6,11}\left(X_{17}\right)=1$. As in the previous proofs, we see that $M^{17}=X_{6} X_{11}+Y$ where $Y$ fibers over $S^{4}$. Thus $X_{6} X_{11}$ fibers over $S^{4}$.

Combining the above results with those of $\$ 3$ reduces the proof of Theorem 2 to the following:

Proof of Theorem 2.

(i) $(\Rightarrow)$ Trivial by Theorem 1 .

$(\Leftarrow)$ By Propositions 3.1, 3.2 and 4.10-4.13, (and the fact that the classes fibered over $S^{4}$ form an ideal), any class in $\mathfrak{R}_{2 n+1}$ is of the form $\omega=\alpha+\beta$ where $\alpha$ fibers over $S^{4}$ and $\beta$ is of the form given in Proposition 3.3. Now suppose $\left[\mathrm{Sq}^{r} v_{t}\right]=0 \in A_{0}^{*}(\omega)$ for $t=n, n-1, r>t-4$. By Theorem $1\left[\mathrm{Sq}^{r} v_{t}\right]=0 \in A_{0}^{*}(\alpha)$ for $t=n, n-1, r>t-4$ as well. Thus $\left[\mathrm{Sq}^{r} v_{t}\right]=0 \in A_{0}^{*}(\beta)$ for $t=n, n-1, r>t-4$ and hence by Proposition $3.3 \beta=0$. Therefore $\omega$ fibers over $S^{4}$.

(ii) By Propositions 3.1, 3.2 and 4.10-4.13, any class in $\mathfrak{R}_{2 n}$ is of the form $\omega=\alpha+\beta$ where $\alpha$ fibers over $S^{4}$ and $\beta$ is of the form given in 
Proposition 3.4. As in (i) we get that $\left[\mathrm{Sq}^{r} v_{t}\right]=0 \in A_{0}^{*}(\beta)$ for $t=n, n-1$, $r>t-4$ and hence by Proposition 3.4 and the remark following it, $\beta=a X^{n-5}\left(X_{4} X_{6}+X_{5}^{2}\right)$. The result follows.

5. A note on fiberings over $\mathbf{S}^{3}$. In [5], it was shown that a complex cobordism class $\omega \in \Omega_{*}^{u}$ fibers over $S^{3}$ if and only if it fibers over $S^{4}$. Here we offer the following

Conjecture 5.1. A class $\omega \in \mathfrak{N}_{2 n}$ fibers over $S^{3}$ if and only if it fibers over $S^{4}$.

We note that by Stong's result (1.1), one direction above is trivial. With regards to the other direction, we have

THEOREM 3. If $\omega \in \mathfrak{N}_{2 n}$ fibers over $S^{3}$, then

$$
\omega=Y+a \mathbf{R} P(2)^{n-5}\left(X_{4} X_{6}+X_{5}^{2}\right)
$$

where $Y$ fibers over $S^{4}$ and $a \in \mathbf{Z}_{2}$.

Proof. By Propositions 3.1, 3.2 and 4.10-4.13, any class in $\mathfrak{R}_{2 n}$ is of the form

$$
\begin{aligned}
\omega= & Y+a_{1} X_{2}^{n}+a_{2} X_{2}^{n-2} X_{4}+a_{3} X_{2}^{n-3} X_{6}+a_{4} X_{2}^{n-5} X_{4} X_{6} \\
& +a_{5} X_{2}^{n-5} X_{5}^{2}, \quad a_{i} \in \mathbf{Z}_{2},
\end{aligned}
$$

where $Y$ fibers over $S^{4}$. Now, if $\omega$ fibers over $S^{3}$, then by Theorem 1 and the $\mathrm{Wu}$ formula we have that all the Stiefel-Whitney numbers of $\omega$ divisible by $w_{2 n}, w_{2 n-1}$ and $w_{2 n-2}$ must be zero.

Claim. All numbers divisible by $w_{2 n-3}$ must be zero as well.

Proof.

(i)

$$
\begin{array}{rlrl}
w_{1}^{3} w_{2 n-3}(\omega) & =w_{1}^{3}\left(\mathrm{Sq}^{n-3} v_{n}+\mathrm{Sq}^{n-2} v_{n-1}\right)(\omega) & & \text { by the Wu formula } \\
& =w_{1}^{3} \mathrm{Sq}^{n-3} v_{n}(\omega) & & \text { by Theorem } 1 \\
& =w_{1}^{2} \cdot \mathrm{Sq}^{1} \mathrm{Sq}^{n-3} v_{n}(\omega) & & \text { since } w_{1}^{3}=w_{1}^{2} \mathrm{Sq}^{1} \\
& =0 & & \text { by the Adem relations } \\
& & \text { and Theorem } 1 .
\end{array}
$$

(ii) Similarly,

$$
w_{3} w_{2 n-3}(\omega)=0 \quad \text { since } w_{3}=w_{2} \mathrm{Sq}^{1} .
$$


(iii)

$$
\begin{aligned}
& w_{1} w_{2} w_{2 n-3}(\omega)=w_{1} w_{2} \operatorname{Sq}^{n-3} v_{n}(\omega) \\
& =\mathrm{Sq}^{3} \mathrm{Sq}^{n-3} v_{n}(\omega) \\
& \text { as in (i) } \\
& = \begin{cases}0 & n=4 k+1, \\
\operatorname{Sq}^{n} v_{n}(\omega), & n=4 k+3, \\
\operatorname{Sq}^{n-1} \operatorname{Sq}^{1} v_{n}(\omega), & n \text { even, }\end{cases} \\
& \text { since } w_{1} w_{2}=v_{3} \\
& =0 \\
& \text { by Theorem } 1 \text {. }
\end{aligned}
$$

This completes the proof of the claim.

Now as in the proof of Proposition 3.4, we apply Stong's result and get that in (5.2) $a_{1}=a_{2}=a_{3}=0$. Finally we note that if $\omega$ fibers over $S^{3}$ or $S^{4}$ then $w_{4} \operatorname{Sq}^{n-3} v_{n-1}(\omega)=0$ by Theorem 1. Invoking (3.5), we get that $a_{4}=a_{5}$ and the result follows.

\section{REFERENCES}

[1] E. H. Brown, Jr., and F. P. Peterson, Relations among characteristic classes I, Topology, 3 (1964), suppl. 1, 39-52.

[2] R. L. W. Brown, Cobordism and bundles over spheres, Michigan Math. J., 16 (1969), 315-320.

[3] P. E. Conner and E. E. Floyd, Fibering within a cobordism class, Michigan Math. J., 12 (1965), 33-47.

[4] A. Didierjean, Classes de cohomologie invariantes par cobordisme et classes de cobordisme se fibrant sur des spheres, Comptes Rendue Acad. Sc. Paris, 289 (1979), 165-167.

[5] S. M. Kahn, Fibering complex manifolds over $S^{3}$ and $S^{4}$, Illinois J. Math., 26 (1982), 296-316.

[6] J. W. Milnor and J. D. Stasheff, Characteristic Classes, Princeton University Press, Princeton, New Jersey, 1974.

[7] D. F. X. O’Reilly, Cobordism classes of fiber bundles, Pacific J. Math., 69 (1977), 467-475.

[8] R. E. Stong, On fibering of cobordism classes, Trans. Amer. Math. Soc., 178 (1973), 431-447.

Received September 20, 1982.

Wayne State University

DETROIT, MI 48202 



\section{PACIFIC JOURNAL OF MATHEMATICS EDITORS}

\author{
DONALD BABBITT (Managing Editor) \\ University of California \\ Los Angeles, CA 90024 \\ J. DugundJI \\ University of Southern California \\ Los Angeles, CA 90089-1113 \\ R. FINN \\ Stanford University \\ Stanford, CA 94305 \\ HERMANN FLASChKa \\ University of Arizona \\ Tucson, AZ 85721
}

C. C. MOORE

University of California

Berkeley, CA 94720

Arthur Ogus

University of California

Berkeley, CA 94720

Hugo RossI

University of Utah

Salt Lake City, UT 84112

H. SAMELSON

Stanford University

Stanford, CA 94305

ASSOCIATE EDITORS
R. ARENS
E. F. BECKENBACH
B. H. NeUmanN
F. WOLF
K. YosHIDA (1906-1982)

\section{SUPPORTING INSTITUTIONS}
UNIVERSITY OF ARIZONA
UNIVERSITY OF BRITISH COLUMBIA
CALIFORNIA INSTITUTE OF TECHNOLOGY
UNIVERSITY OF CALIFORNIA
MONTANA STATE UNIVERSITY
UNIVERSITY OF NEVADA, RENO
NEW MEXICO STATE UNIVERSITY
OREGON STATE UNIVERSITY

\author{
UNIVERSITY OF OREGON \\ UNIVERSITY OF SOUTHERN CALIFORNIA \\ STANFORD UNIVERSITY \\ UNIVERSITY OF HAWAII \\ UNIVERSITY OF TOKYO \\ UNIVERSITY OF UTAH \\ WASHINGTON STATE UNIVERSITY \\ UNIVERSITY OF WASHINGTON
}

The Supporting Institutions listed above contribute to the cost of publication of this Journal, but they are not owners or publishers and have no responsibility for its content or policies.

Mathematical papers intended for publication in the Pacific Journal of Mathematics should be in typed form or offset-reproduced (not dittoed), double spaced with large margins. Please do not use built up fractions in the text of the manuscript. However, you may use them in the displayed equations. Underline Greek letters in red, German in green, and script in blue. The first paragraph must be capable of being used separately as a synopsis of the entire paper. In particular it should contain no bibliographic references. Please propose a heading for the odd numbered pages of less than 35 characters. Manuscripts, in triplicate, may be sent to any one of the editors. Please classify according to the scheme of Math. Reviews, Index to Vol. 39. Supply name and address of author to whom proofs should be sent. All other communications should be addressed to the managing editor, or Elaine Barth, University of California, Los Angeles, California 90024.

There are page-charges associated with articles appearing in the Pacific Journal of Mathematics. These charges are expected to be paid by the author's University, Government Agency or Company. If the author or authors do not have access to such Institutional support these charges are waived. Single authors will receive 50 free reprints; joint authors will receive a total of 100 free reprints. Additional copies may be obtained at cost in multiples of 50 .

The Pacific Journal of Mathematics is issued monthly as of January 1966. Regular subscription rate: $\$ 190.00$ a year (5 Vols., 10 issues). Special rate: $\$ 66.00$ a year to individual members of supporting institutions.

Subscriptions, orders for numbers issued in the last three calendar years, and changes of address should be sent to Pacific Journal of Mathematics, P.O. Box 969, Carmel Valley, CA 93924, U.S.A. Old back numbers obtainable from Kraus Periodicals Co., Route 100, Millwood, NY 10546.

The Pacific Journal of Mathematics at P.O. Box 969, Carmel Valley, CA 93924 (ISSN 0030-8730) publishes 5 volumes per year. Application to mail at Second-class postage rates is pending at Carmel Valley, California, and additional mailing offices. Postmaster: Send address changes to Pacific Journal of Mathematics, P.O. Box 969, Carmel Valley, CA 93924.

PUBLISHED BY PACIFIC JOURNAL OF MATHEMATICS, A NON-PROFIT CORPORATION

Copyright $\odot 1984$ by Pacific Journal of Mathematics 


\section{Pacific Journal of Mathematics}

Vol. 114, No. $2 \quad$ June, 1984

William Allen Adkins, A Harnack estimate for real normal surface

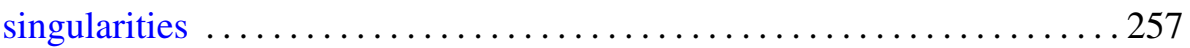

George E. Andrews, Multiple series Rogers-Ramanujan type identities . . . . 267

Didier Arnal, $*$ products and representations of nilpotent groups . . . . . . 285

David Cox and Walter Raymond Parry, Representations associated with

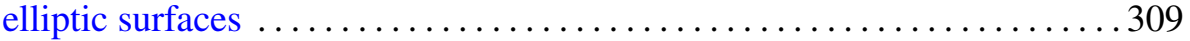

Joanne Marie Dombrowski, Tridiagonal matrix representations of cyclic

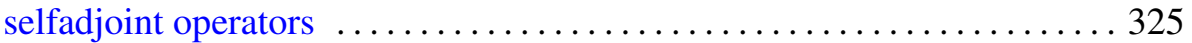

Ronald Dotzel, An Artin relation $(\bmod 2)$ for finite group actions on

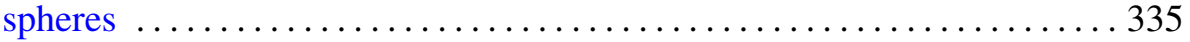

Leo Egghe, Convergence of adapted sequences of Pettis-integrable

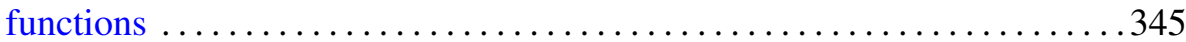

Rebecca A. Herb, Characters of induced representations and weighted

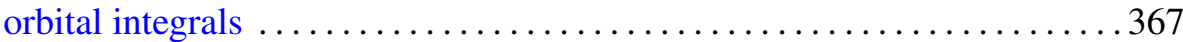

Steven M. Kahn, Cobordism obstructions to fibering manifolds over

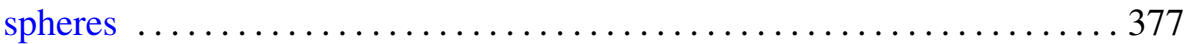

Robert D. Little, Projective space as a branched covering of the sphere with orientable branch set

Claude Schochet, Topological methods for $C^{*}$-algebras. III. Axiomatic

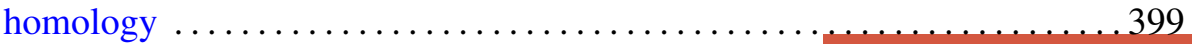

Claude Schochet, Topological methods for $C^{*}$-algebras. IV. $\bmod p$ homology

James M. Stormes, On the $K O$-orientability of complex projective varieties

Josephine Anne Ward, Characterization of homogeneous spaces and their norms 